\title{
Elasticity and relaxation in full and partial vitrimer networks
}

\author{
Fanlong Meng, ${ }^{\dagger}$ Mohand O. Saed, ${ }^{\ddagger}$ and Eugene M. Terentjev*, \\ $\dagger$ †Max Planck Institute for Dynamics and Self-Organization, Göttingen 37077, Germany \\ $\ddagger$ Cavendish Laboratory, University of Cambridge, JJ Thomson Avenue, Cambridge CB3 \\ OHE, U.K. \\ E-mail: emt1000@cam.ac.uk
}

\begin{abstract}
We develop a continuum model of dynamic-mechanical response of vitrimers, where the elastic energy of the material accounts for the conserved number of the crosslinks in the network. We also prepare partial vitrimer networks, which consist of variable fractions of transient network based on boronic ester bond-exchange, and of a permanent polymer network. By fitting the theory to our experimental data on stress relaxation, the bond-exchange rate and the fraction of the permanent elastic network are obtained with a linear relationship between the fraction of the transient polymer network and the ratio between the boronic ester and the flexible spacer among the chain-extending thiols. For a 100\% vitrimer undergoing a ramp deformation, the stress of the material first increases and then decreases, where the yield time decreases with the increasing strain rate. A partial vitrimer can behave as a pure elastic material without yielding at low strain rates or show a non-monotonic 'S-shaped' stress-strain relationship at high strain rates.
\end{abstract}




\section{Introduction}

Transient polymer networks can be categorised as physically or chemically bonded, depending on the type of the crosslinks in the materials. $\frac{112}{2}$ In a physical transient network, ${ }^{3}$ the crosslinks are formed by various physical interactions between polymer chains, such as hydrogen bonds,,$\frac{45}{}$ ionic interactions,,$\underline{6}$ or hydrophobicity. ${ }^{7}$ The crosslinks in chemical tran-

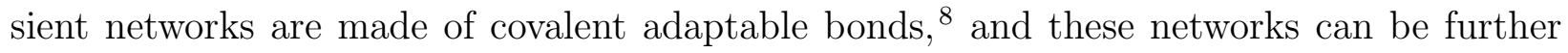
divided into two sub-classes: dissociative and associative. In dissociative transient networks, a crosslink first breaks and then re-forms at another place, while in the associative case, an original crosslink only breaks after a new crosslink is formed. The term 'vitrimers' has been first introduced in the work of Leibler et al., $\stackrel{910}{ }$ referring to a subset of covalently adaptable networks that can change its topology while maintaining the constant number of chemical bonds. Such networks with significant capacity for the bond-exchange reaction (BER) have attracted a lot of attention as a new class of associative chemical transient networks, as reviewed in Ref.11 In vitrimers, the covalently crosslinked polymer chains can dynamically exchange, when activated by a catalyst and/or elevated temperature. The re-organisation of vitrimer network structure has a generic analogy with the silicate glass, which has the same bonds between the $\mathrm{SiO}_{2}$ units remaining above the vitrification temperature (unlike thermoplastic polymer, colloid or metallic glass that become liquid at high temperature due to the loss of bond connectivity), with only the rate of exchange increasing rapidly, promoting plastic flow. A number of reaction strategies, such as transesterification, ${ }^{9|12| 13}$ transamination of vinylogous urethanes, $\frac{14}{14}$ transalkylation, ${ }^{15[16}$ disulfide exchange, $\frac{17 \mid 18}{\operatorname{tran}}$ scarbamoylation $^{19}$ have been employed to form covalent bond-exchangeable networks. As a result of their strictly conserved number of covalent bonds, vitrimers can possess excellent mechanical properties of typical thermosets at low temperatures, but also demonstrate high processbility at high temperatures as thermoplastics.

Recently, the concept of boronic cross-coupling (originally studied in small molecule synthesis ${ }^{20 \mid 21}$ ) has been applied to polymeric systems. ${ }^{22}$ Unlike other BER systems, boronic 
exchanges do not generally need a catalyst, initiator, or elevated temperature. The readiness of boronic bond exchange strongly depends on the nature of the polymer network that encompasses these bonds (e.g. hydrophilic, hydrophobic, glassy, semi-crystalline or rubbery). For example, in a hydrophilic network the boronic bond exchange can occur via hydrolysis at low temperature, wheras in highly hydrophobic and semi-crystalline networks the bond exchange can only occur at temperature above melting. 23124

Physically-bonded transient networks are well understood theoretically. $25 \mid 31$ Here the crosslink number is not constant, and so the breakage and the re-forming of the crosslinks are treated as two independent processes. The characteristic property of vitrimers, that the number of covalent bonds remains constant at all times, not only helps to maintain the integrity of the material at a high temperature, but also keeps the effect of non-affine deformation almost unchanging during deformation, as the non-affine effects are usually local and only depend on the networks connectivity, which is strictly conserved in vitrimers. However, a continuum theory of vitrimers incorporating this distinguishable property is still absent, apart from some initial theoretical discussions on the material rheology based on the statistical viewpoint ${ }^{[32}$ and on material entropy and viscosity by simplifying vitrimers as patchy particle networks,, 33 as well as numerical attempts by molecular dynamics simulation. $\frac{34135}{3}$

Here we theoretically derive a model continuum free energy of vitrimers under deformation, where the constraint of constant crosslink number is implemented. It turns out that the

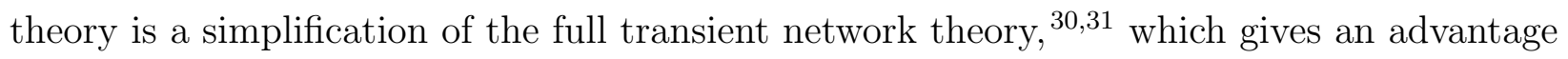
of presenting some closed analytical expressions for constitutive relations. We also extend the theory to include partial vitrimer networks: a situation when a certain fraction $\nu$ of the network is permanently crosslinked, while the remaining $(1-\nu)$ is connected by BER-capable bonds. Based on the general theory, we discuss two practical cases: of the stress relaxation of a vitrimer after an instantaneous deformation, and of the stress-strain response under a constant ramp deformation, for both full and partial vitrimers. The theoretical results are compared with our experiments on a fully exchangeable vitrimer network, and also a network 
with partial boronic-exchange segments and partially permanent strands.

\section{Theoretical Section}

Let us consider polymer network strands linked by bonds that can be broken with a characteristic rate (chain exchanging rate):

$$
\beta=\omega_{0} e^{-W_{c} / k_{B} T}
$$

Here $\omega_{0}$ is the rate of attempt for this exchange reaction, which is a constant about $10^{8}$ $10^{9} \mathrm{~s}^{-1}$ for ordinary small molecules in thermal motion ${ }^{36}$ (in crowded systems and for larger fluctuating molecules it could be as low as $10^{6} \mathrm{~s}^{-1}$ ). The energy barrier of bond exchange is $W_{c}$, and $k_{B} T$ is the thermal energy. Here for seeking the transparency of the theory, the chain exchanging rate is treated independent of the tensile forces acting on the network strands, which is valid when the forces acting on the polymer strands are small (typically in the case where the contour length of the strand is much larger than the distance between two neighboring crosslinks). Note that a significant force dependence of the breakage rate can alter the material's responses, such as inducing the stretched exponential stress relaxation, for which a detailed discussion can be found in previous works. $\frac{30131}{3 n}$ vitrimers the chains broken from the crosslinks are immediately linked back into the network in the force-free configuration, so the total number of crosslinks is conserved.

The local state of a permanently crosslinked elastic material without any mechanic load is defined as its reference state. Consider an unloaded network consisting of $N_{0}$ crosslinked chains at time $t=0$. After an infinitesimal time interval $\Delta t$, the number of crosslinked chains crosslinked at $t=0$ will decrease to $N_{0}(1-\beta \Delta t) \simeq N_{0} e^{-\beta \Delta t}$. Simultaneously, some of the chains that have been made free during this interval of time will be recrosslinked back into the network, assumed in a relaxed zero-force configuration, and the number of those is identical to that of the chains broken from the crosslinks: $N_{0} \beta \Delta t$. After the next time 
interval $\Delta t$, i.e. at $t=2 \Delta t$, there will be three types of crosslinked chains in the networks; chains originally crosslinked at $t=0$ with their number decreasing further to $N_{0} e^{-2 \beta \Delta t}$, chains recrosslinked at $t=\Delta t$ with their number decreasing to $N_{0} \beta \Delta t e^{-\beta \Delta t}$, and finally chains newly recrosslinked in the zero-force relaxed topology within this time interval from $\Delta t$ to $2 \Delta t$, with their number being $N_{0} \beta \Delta t$. By iterating the above process, the total number of crosslinked chains at a given time $t$ can be expressed in a continuous form:

$$
N(t)=N_{0}\left[e^{-\beta t}+e^{-\beta t} \int_{0}^{t} \beta e^{\beta t^{\prime}} d t^{\prime}\right]
$$

One can identify that the expression in square brackets is identically equal to 1 , which confirms that the total number of crosslinked chains is conserved in vitrimers. Correspondingly, the energy density of a vitrimer is expressed as a combination of two terms:

$$
F_{v}(t)=\underbrace{e^{-\beta t} F_{p}(t ; 0)}_{(1)}+\underbrace{e^{-\beta t} \int_{0}^{t} \beta e^{\beta t^{\prime}} F_{p}\left(t ; t^{\prime}\right) d t^{\prime}}_{(2)} .
$$

The first term on the right-hand-side represents the contribution from the crosslinked chains surviving from the beginning $(t=0)$ until the current time $t$, while the second term represents the elastic contributions from the chains re-crosslinked at different times $t^{\prime}$ during the deformation process, for which the dynamic deformation state at $t^{\prime}$ represents the new reference state. In the equation $(3), F_{p}\left(t ; t^{\prime}\right)$ is the elastic energy of the network at a time $t$, which is deformed with respect to the reference state at a time $t^{\prime}$; the reference states can only be defined dynamically, according to when polymer chains are re-crosslinked. Note that although the re-crosslinked polymer chains may contribute some elastic energy due to a small residual tension, however, the contribution would be small compared with those crosslinked from the beginning and can only change the result quantitatively; $\frac{37}{37}$ thus we assume the reference state of newly re-crosslinked polymer chains for simplicity. Although other expressions accounting for large deformation are available, $\stackrel{37}{\text { in }}$ this work we adopt the simplest 
neo-Hookean model for $F_{p}\left(t ; t^{\prime}\right)$, which is valid up to a medium-magnitude deformations (a straightforward extension for high strains is possible if and when needed):

$$
F_{p}\left(t ; t^{\prime}\right)=\frac{1}{2} G\left\{\operatorname{tr}\left[\mathbf{E}^{T}\left(t ; t^{\prime}\right) \mathbf{E}\left(t ; t^{\prime}\right)\right]-3\right\}
$$

where $G$ is the rubber (shear) modulus of the network, in the ideal rubber purely determined by the crosslinking density, $G=n_{x} k_{\mathrm{B}} T$, and the deformation tensor at time $t$ with respect to the references state at time $t^{\prime}$ is defined as $\mathbf{E}\left(t ; t^{\prime}\right)=\mathbf{E}(t ; 0) \mathbf{E}^{-1}\left(t^{\prime} ; 0\right)$.

For the partially vitrimer network, there will be a certain amount of permanently crosslinked chains that never undergo the bond exchange (and the associated reduction of tensile force on the polymer strands). If we denote the fraction of such permanent network strands as $\nu$, then the energy density of a partial vitrimer will be modified to:

$$
F_{\text {partial }}(t)=\left[\nu+(1-\nu) e^{-\beta t}\right] F_{p}(t ; 0)+(1-\nu) e^{-\beta t} \int_{0}^{t} \beta e^{\beta t^{\prime}} F_{p}\left(t ; t^{\prime}\right) d t^{\prime}
$$

\section{Experimental Section}

As an example system to test our theory, we have prepared several rubbery networks of endlinked flexible polymer chains formed by an alternating di-vinyl and di-thiol units, crosslinked by the fixed amount $(30 \mathrm{~mol} \%)$ of 4 -functional flexible thiols. $\frac{38}{3}$ The vitrimer property is added to the network via the boronic ester monomers, prepared following the methods of Chen et al., ${ }^{39}$ which have been shown to have a fast catalyst-free exchange reaction when the -BOO group on one chain switches with a matching group on another chain in close

proximity. $\stackrel{23 / 40141}{ }$ Figure 1 illustrates the relevant chemistry and gives the nomenclature of different samples, the final synthesis following the methods reported in. $\frac{38 \mid 42}{10}$ The $100 \mathrm{bdb}$ sample has a very high fraction of exchanging bonds and should be readily turning into a plastic regime; in contrast, at low concentration of boronic ester groups the high fraction of the network is made of permanently bonded chains. 
(a)

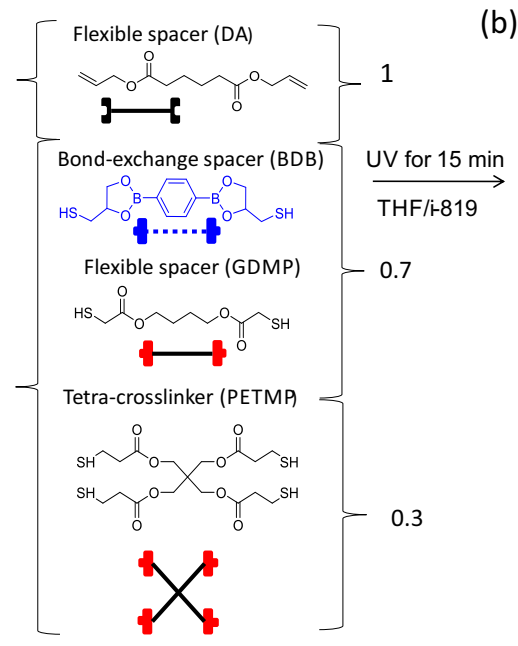

(b)
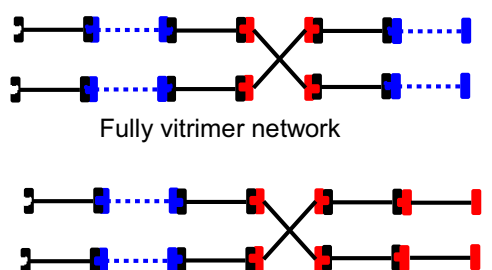

Partially vitrimer network

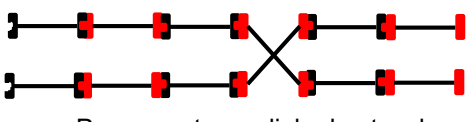

(c)

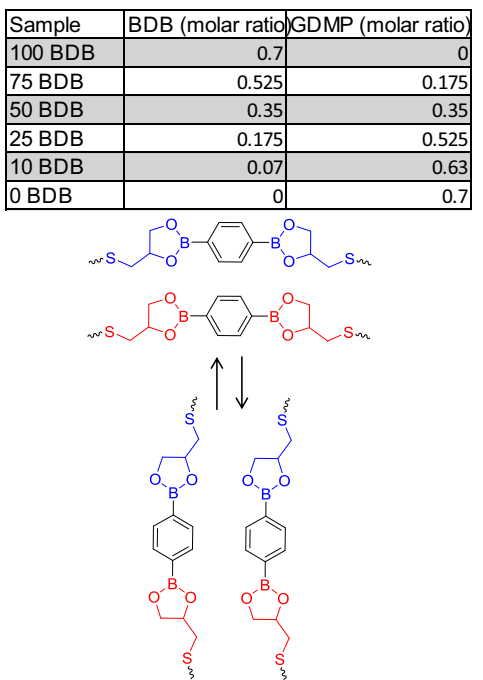

Figure 1: Chemical composition and reaction schematic of thiol-ene vitrimers: (a) We use the 1:1 stoichiometric ratio of vinyl and thiols (1.0 divinyl: 0.7 dithiol: 0.3 tetrathiol); the UV-initiated 'click' reaction between the vinyl $\mathrm{C}=\mathrm{C}$ bond and the thiol $-\mathrm{SH}$ group proceeds to a near- $100 \%$ completion in our mixture. The di-vinyl flexible chain extenders alternate with di-thiol chain extenders (with the fixed 30\% molar ratio of the 4-functional flexible crosslinker). However, different samples have a different proportion between the plain flexible di-thiol chain extender, and the boronic ester thiol that is capable of fast bond-exchange reaction. (b) The scheme of our networks that may have all network strands containing the exchangeable boronic groups (labelled by dashed segments), or a fraction of permanent strands (labelled by solid segments), or a fully permanent network. (c) The table labels the samples by the fraction of the boronic ester among the total of chain-extending thiols. (d) The scheme of boronic ester bond exchange.

The modern 'click' chemistry $\underline{43}$ is a robust and reliable approach, the reaction between the vinyl $\mathrm{C}=\mathrm{C}$ bond and the thiol $-\mathrm{SH}$ group proceeds to a near full completion with an exact

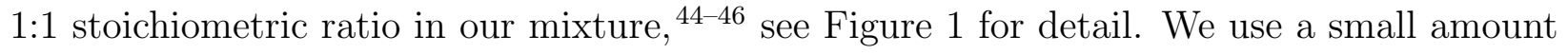
(20 wt\%) of tetrahydrofuran (THF) solvent to dilute the crystalline boronic ester di-thiol (all other molecules are liquid at room temperature) and $2 \mathrm{wt} \%$ of UV photoinitiator (Irgacure 819). The UV-stimulated reaction is complete in ca.10 minutes at room temperature, after which we remove the residual THF in the vacuum oven at $80^{\circ} \mathrm{C}$ overnight.

Standard tests of gel fraction were carried out to test the reaction conversion and the network integrity: we used the 0bdb sample without any boronic ester groups, because the exposure of these exchangeable bonds to solvent is strongly affected by the residual 
humidity $\stackrel{24 \mid 40}{2}$ and could produce artefacts in the gel fraction. The 0bdb network was swollen to a maximum of $34 \pm 2 \%$ after 3 days in toluene, and of $169 \pm 3 \%$ after 3 days in THF. After drying for 3 days at $80^{\circ} \mathrm{C}$ in the vacuum oven, we have recovered $90.4 \pm 0.2 \%$ of the sample weight in toluene, and $87.4 \pm 0.4 \%$ of the sample weight in THF. This confirms the reliability of the 'click' chemistry involved in making our networks.

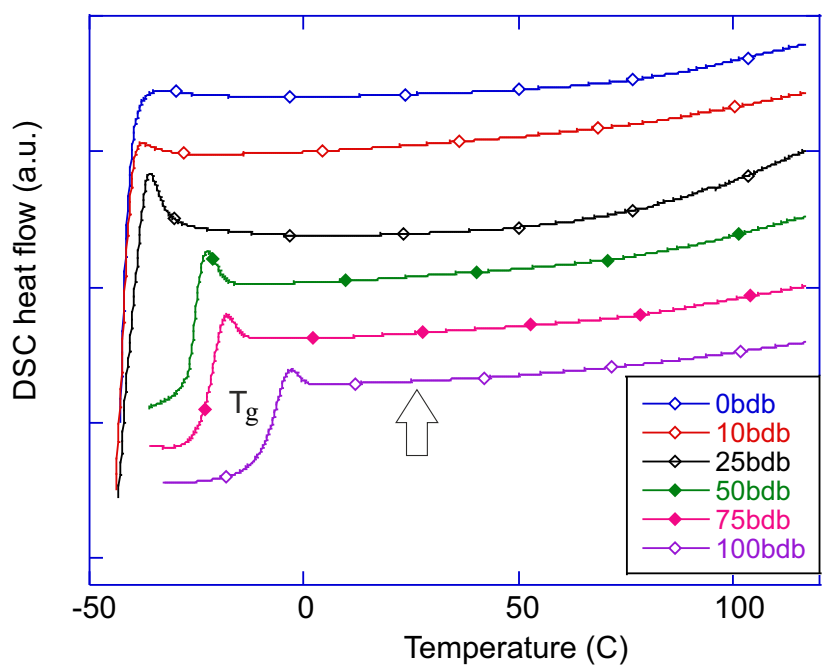

Figure 2: The differential scanning calorimetry (DSC) data on all the samples (in heating at $10^{\circ} \mathrm{C} / \mathrm{min}$ ), showing the glass transition $T_{g}$ below zero (shifting down to $-40^{\circ} \mathrm{C}$ on decreasing fraction of boronic ester, which is a more rigid molecular moiety leading to a stronger glass propensity). The main point of this plot is to illustrate that our mechanical measurements are done safely far from the glass transition: the arrow points where the relaxation and ramp experiments were carried out.

We used the differential scanning calorimetry (Perkin Elmer DSC 4000) to characterize the phase sequences in all samples. Figure 2 illustrates the lack of internal transitions in any of the networks, apart from the glass transition that is safely below $0^{\circ} \mathrm{C}$ in all cases. There is an evident shift of the lower glass transition temperature when the more flexible DA chain extenders replace the more conjugated BDMP, however, our main concern was that in all mechanical tests we are working with a classical rubbery network at the fixed ambient temperature of $20^{\circ} \mathrm{C}$, for which the neo-Hookean elastic energy is a good first approximation. All samples were characterized by a dynamic-mechanical frequency scan (at constant $T=$ $20^{\circ} \mathrm{C}$ ), using the Metravib VA 4000, confirming that there was no trace of a dynamic glass 
transition (a peak of internal relaxation and loss factor $\tan \delta$ ) at frequencies below $450 \mathrm{~Hz}$. Main mechanical tests were carried out in tensile mode, using a custom-built apparatus that was designed to impose an isothermal constant rate of strain, including the zero-rate (fixed strain) for the stress relaxation measurements shown in Figure 3.

\section{Results and Discussions}

For an incompressible material undergoing a uniaxial stretch along $\boldsymbol{e}_{x}$ direction with a stretch ratio $\lambda(t)$, the deformation tensor $\mathbf{E}(t ; 0)$ has principal values of $\lambda(t)$ along $\boldsymbol{e}_{x}$, and $1 / \sqrt{\lambda(t)}$ along both $\boldsymbol{e}_{y}$ and $\boldsymbol{e}_{z}$. Then the neo-Hookean energy density will simply become $F_{p}(t ; 0)=$ $\frac{1}{2} G\left[\lambda(t)^{2}+2 / \lambda(t)-3\right]$.

(1) Stress relaxation. When an instantaneous tensile deformation of stretch ratio $\lambda$ is imposed at $t=0$, the energy of the vitrimer will have no contribution from the chains re-crosslinked after $t=0$ because their current deformation state at $t^{\prime}<t$ is identical with their reference state defined by the stretch ratio $\lambda$ along $\boldsymbol{e}_{x}$ direction. Then the tensile stress of the stretched partial vitrimer network can be written as

$$
\sigma(t)=\left[\nu+(1-\nu) e^{-\beta t}\right] \frac{\partial}{\partial \lambda} F_{p}(t ; 0)=G\left(\lambda-1 / \lambda^{2}\right)\left[\nu+(1-\nu) e^{-\beta t}\right]
$$

This stress decays with time in the simple-exponential form, which is the key characteristic of a vitrimer possessing a unique energy barrier for the chain exchanging (as opposed to a physically bonded transient network, where the broad distribution of the energy barrier for the crosslink breakage leads to a slower stretched-exponential decay $\sqrt[30 \mid 47 / 48]{ })$. However, vitrimers could also exhibit a stretched exponential stress relaxation: the bond-exchange rate may have a distribution due to a tensile force dependence of the connecting two crosslinks. 31 The force dependence of chemical reaction rate is the essence of 'mechanochemistry', and it is not a frequent occurrence in practice. However, in our case, this could explain the slight deviations of the theoretical simple-exponential curve from the experimental data in 
Figure 3(a), whereas an almost perfect fitting can be obtained with a stretched exponential function with the stretching factor as 0.7 (other fitting parameters, $\nu$ and $\beta$, remaining almost unchanged). The stretch factor 0.7 is much larger than that of physical transient networks, where a stretch factor of 0.2 was reported for styrene-isoprene-styrene thermoplastic elastomers in our previous work. ${ }^{30 / 49}$ The stress relaxation ends with a residual stress $\nu G\left(\lambda-1 / \lambda^{2}\right)$.

The relaxation was tested in our vitrimers after a fast imposition of a constant strain (a step of $3 \%$ ), at constant temperature of $20^{\circ} \mathrm{C}$. Figure 3(a) shows that the samples with high vitrimer content $(>30 \mathrm{~mol} \%)$ are relaxing their tensile stress to zero within a few seconds (the decay rate $\sim 0.3 \mathrm{~s}^{-1}$ in Table 1), via what is essentially a plastic flow. By decreasing the amount of the boronic ester (fraction $<30 \mathrm{~mol} \%$ ), there is an increasing amount of permanent percolating network in the samples, which shows in the stable equilibrium tensile modulus. For a rubber network without any exchangeable boronic ester (0bdb), the stress remains almost a constant (decay rate below $0.01 \mathrm{~s}^{-1}$ ). Note that for vitrimers of moderate amount of boronic ester, for example the sample labelled 25bdb, the relaxation rate of the stress appears slightly higher than that of the vitrimer containing more boronic ester, which can be explained by the increasing local stress from the overstretched permanent fraction of the network. In other words, permanent stress facilitates the stress relaxation of vitrimers by maintaining internal tension. Moreover, the fraction of the percolating permanent network in a vitrimer $\nu$ decreases in an almost linear manner, with $\nu=1-3.3 \cdot p_{\mathrm{bdb}}$, which becomes zero for $p_{\mathrm{bdb}}>30 \mathrm{~mol} \%$ suggesting the network percolation threshold, see Figure $3(\mathrm{~b})$.

Table 1: Fitted fractions of non-exchangeable (permanent) network and exchange rates for vitrimers of different bdb fractions.

\begin{tabular}{c|c|c}
\hline bdb fraction (\%) & fraction $\nu$ & exchange rate $\beta\left(\mathrm{s}^{-1}\right)$ \\
\hline \hline 0 & $0.98_{+}$ & $0.01_{-}$ \\
\hline 10 & 0.59 & 0.57 \\
\hline 25 & 0.22 & 0.35 \\
\hline 50 & 0.03 & 0.30 \\
\hline 100 & 0.02 & 0.35 \\
\hline
\end{tabular}




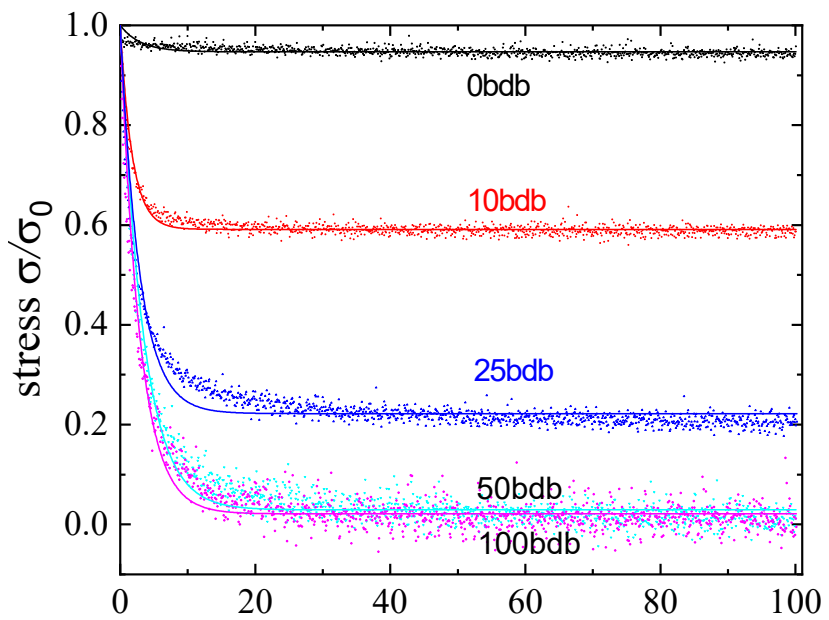

(a)

time (s)

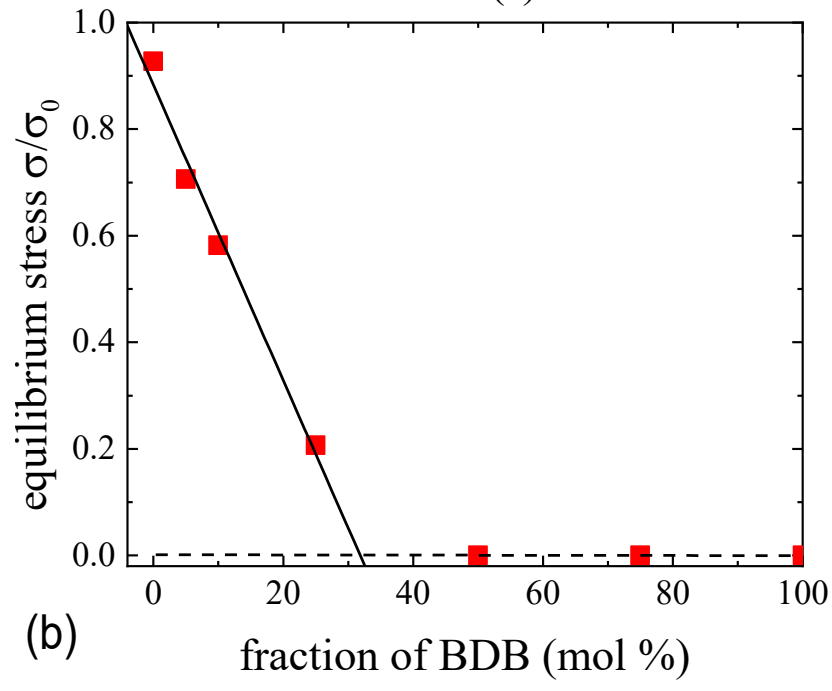

Figure 3: (a) Stress relaxation of vitrimers under the constant uniaxial strain of $3 \%$ imposed as a step at $t=0$, for the range of networks with the fraction of permanent component labelled on the plot. Solid lines are obtained by fitting from Eq. (6) with fitting parameters listed in Table1. (b) The fraction of the permanent network in a vitrimer from Table 1, [or the residual equilibrium modulus in the networks with varying fraction of vitrimer component [plotting the final saturation values of the relaxation curvs in (a)] against the fraction of boronic ester in the network strands. 
(2) Ramp deformation. Consider a vitrimer uniaxially stretched with a constant rate $\dot{\varepsilon}$, so that the uniaxial stretch ratio is increasing with time: $\lambda(t)=1+\dot{\varepsilon} t$. The stress of the vitrimer, $\sigma=\partial F_{\text {partial }} / \partial \lambda$, can be obtained as an integral expression:

$$
\frac{\sigma(t)}{G}=\left[\nu+(1-\nu) e^{-\beta t}\right]\left[\lambda(t)-\frac{1}{\lambda(t)^{2}}\right]+(1-\nu) e^{-\beta t} \int_{0}^{t} \beta e^{\beta t^{\prime}}\left[\frac{\lambda(t)}{\lambda\left(t^{\prime}\right)^{2}}-\frac{\lambda\left(t^{\prime}\right)}{\lambda(t)^{2}}\right] d t^{\prime}
$$

For a full vitrimer, i.e. for $\nu=0$, the stress is characteristically non-monotonic: first increasing and then decreasing with the increasing strain as shown by the solid curves in Figure 4. This remains the case even for non-zero (low) fraction of the permanent network $(\nu \neq 0)$. The maximum stress is defined as the yielding point, or the elastic-plastic transition point:

$$
\left.\frac{\partial \sigma(t)}{\partial t}\right|_{\mathrm{y}} \equiv 0
$$

Solving this equation numerically gives the expression for the yield time, or equivalently the yielding strain $\lambda_{\text {yield }}=\dot{\varepsilon} t_{\text {yield }}$, which we examine below.

To test these predicitions experimentally, we used the full vitrimer with $\nu=0$ (the vitrimer network 100bdb in Figure 1). As shown in Figure 5(a), when the strain rate is small compared to the chain exchanging rate of the crosslinks, i.e. $\dot{\varepsilon} / \beta \ll 1$, both the yielding stress $\sigma_{\mathrm{y}}$ and the yielding strain $\lambda_{\mathrm{y}}$ increase with the strain rate $\dot{\varepsilon}$, and the yield time $t_{\mathrm{y}}$ decreases with the strain rate, as illustrated in Figure 5(b) by comparing the experimental values with the theoretical curve from Eq. (8) for the same parameters. The experimental results match well with the theory in this region of low strain rate.

However, there exists a critical strain rate $\dot{\varepsilon}^{c} \simeq 3.7 \beta$, where the yield time is the minimum, equal to $\beta t_{\mathrm{y}} \simeq 0.89$. If the applied strain rate is approaching or larger than this critical $\dot{\varepsilon}^{c}$, then the theoretically calculated yield time $t_{\mathrm{y}}$ increases with increasing strain rate, and eventually saturates at $\beta t_{\mathrm{y}}=1$ for the $\nu=0$ network. In our experiments, the situation can be different depending on how stress relaxation of the network depends on the mechanic 


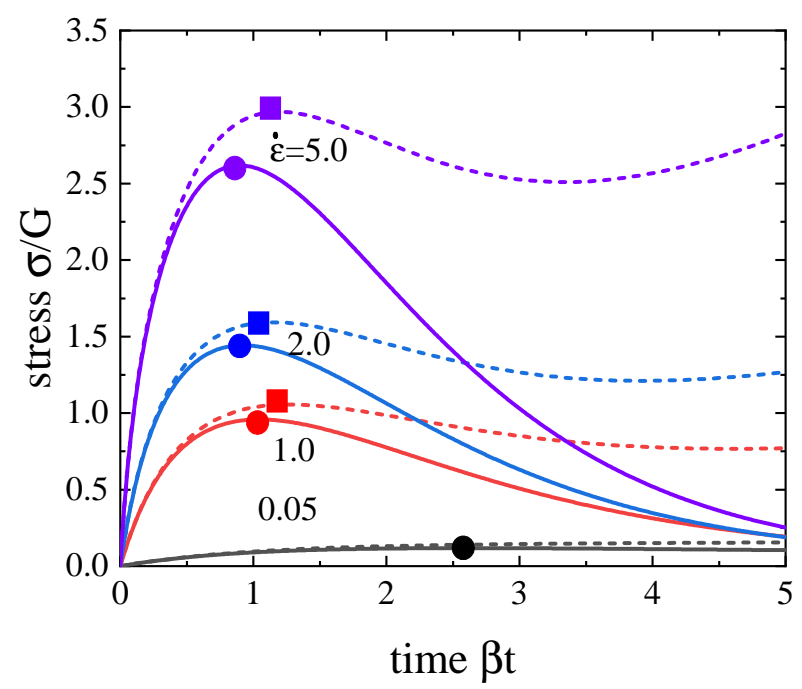

Figure 4: Stress-strain relationship of vitrimers under a ramp stretch numerically calculated from Eq. 7 (solid: $\nu=0.0$ for a full vitrimer, and dashed: $\nu=0.1$ for a partial vitrimer). Circles and squares denote the yielding points of cases of various strain rates for $\nu=0$ and 0.1 , respectively.

properties of the materials. For example, the yield time in Figure 5(b) obtained from our experiment is small compared to the theoretical results. The reason is that, the chain exchanging rate of the crosslinks in a vitrimer can increase with the strain of the material due to the increased forces acting on the polymer chains. When polymer strands in the network are stretched to the limit defined by their finite extensibility, the tensile forces acting on the chains are no longer negligible, and one then needs to take account of the force dependence of the bond exchange rate, and also a change to other continuum elastic models of permanent elastomers accounting the finite stretchability to replace the neo-Hookean expression we take for small deformations, e.g. the Gent model. 50 In the ramp experiment, with the progress of time, the crosslinked chains experience larger deformation, which induces a higher chain exchanging rate. This leads to a faster stress decay, and therefore explains why the experimental stress decays faster than that of the theoretical one after yielding in Figure 5(a). We have neglected this effect to keep the theoretical model simple and analytical, but clearly this approximation is only valid when the imposed strain rates are low. 

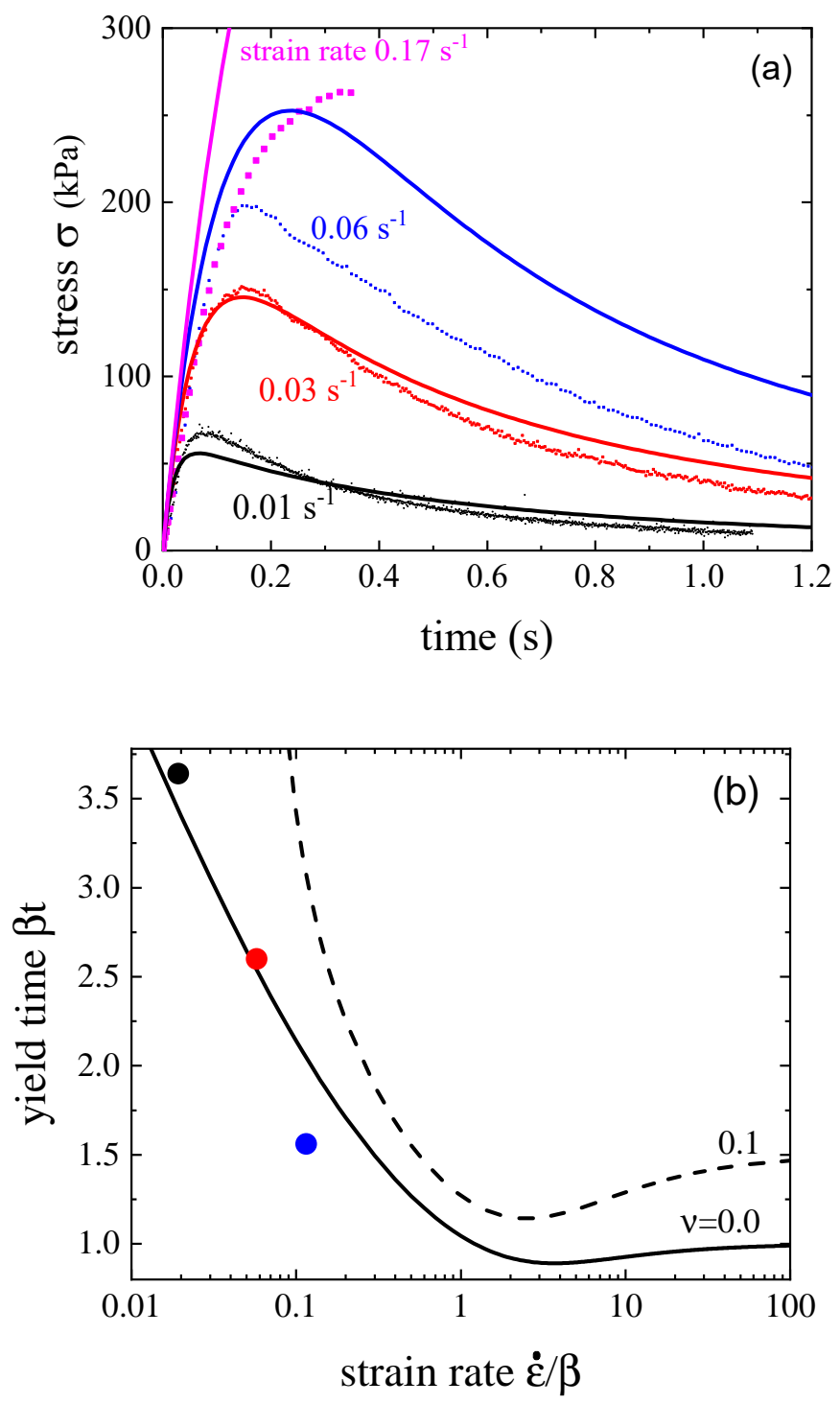

Figure 5: (a) Stress-strain relationship of vitrimers 100bdb under uniaxial stretch of various strain rate, where the dots and the solid lines correspond to experimental and theoretical results, respectively. Fitting parameters are: $G=1100 \mathrm{~Pa}$ and $\beta=0.52 \mathrm{~s}^{-1}$. (b) Yielding time as a function of strain rate (solid and dash lines correspond to $\nu=0$ and 0.1 , as in Figure (4). The dots correspond to the data extracted from the experiments data in plot (a), where the black, red and blue one denote the strain rate $0.01 \mathrm{~s}^{-1}, 0.03 \mathrm{~s}^{-1}$ and $0.06 \mathrm{~s}^{-1}$, respectively. 
In the high strain-rate limit of $\dot{\varepsilon} t \gg \beta t \gg 1$, the tensile stress of the material is obtained from Eq. (7): $\sigma(t) \simeq G e^{-\beta t}(1+\dot{\varepsilon} t)$. The yield time can be easily obtained analytically as: $t_{\mathrm{y}}=(\dot{\varepsilon}-\beta \dot{\varepsilon}) / \beta \simeq 1 / \beta$, which is also identified by the numerically calculated solid curve in Figure 5(b) where the yield time is $t_{\mathrm{y}} \simeq 1 / \beta$ for large strain-rates $\dot{\varepsilon} / \beta \geq 100$.

For a partial vitrimer network with a non-zero $\nu$, the stress-strain relation can be different. Taking a low value of $\nu=0.1$ as an example, if the strain rate is small, then the stress of the material just increases monotonously without showing the decrease, as shown in Figure 4. If the strain rate is large, the stress of the vitrimer becomes a non-monotonic function of strain (which grows lineraly with time), i.e. first increases, and then decreases as that of $\nu=0.0$, but then followed by a second increase in stress due to the permanent network component represented by a non-zero $\nu$. When the material responds with such 'S-shape' stress-strain relations, there can be a neck-like region forming, where there will be a region of a large stretch ratio coexisting with a region of a small stretch ratio. However, this was not observed in our experiment mostly because of the material failure at large stresses before yielding, at high strain rates. Compared with full vitrimers (like our 100bdb material), it takes longer for partial vitrimers to yield, because these systems are stiffer due to the permanent component in the network.

\section{Conclusion}

Vitrimers have been fabricated in various forms with excellent mechanical strength and reprocessibility without losing the integrity of the material. In order to understand the mechanic properties of the vitrimers, we propose a continuum model to describe how the full or partial vitrimers respond upon deformations, by incorporating the polymer chain exchange dynamics. With the model, the stress relaxation of vitrimers upon an instantaneous deformation and the stress-strain relation under a ramp deformation have been discussed as an example case, where the theoretical results match well with our experiments. We believe 
this simple and portable model can provide the quantitative description of the rheology of this wonderful class of materials, as well as serving as the guide for its future fabrications of desired rheological properties and applications.

\section{Acknowledgments}

This work was supported by the European Research Council grant No: 786659. F. M. acknowledges Humboldt Foundation for the support. The authors are grateful for many useful communications and advice from Takuya Ohzono and Alexandra Gablier.

\section{References}

(1) Wei, Z.; Yang, J. H.; Zhou, J.; Xu, F.; Zrínyi, M.; Dussault, P. H.; Osada, Y.; Chen, Y. M. Chem. Soc. Rev. 2014, 43, 8114-8131.

(2) Yang, Y.; Urban, M. W. Chem. Soc. Rev. 2013, 42, 7446-7467.

(3) Seiffert, S.; Sprakel, J. Chem. Soc. Rev. 2012, 41, 909-930.

(4) Stadler, R.; de Lucca Freitas, L. Polym. Bull. 1986, 15, 173-179.

(5) Stadler, R. Permanent and transient networks; Steinkopff: Darmstadt, 1987; Vol. 75; pp $140-145$.

(6) MacKnight, W. J.; Earnest, T. R. J. Polym. Sci.: Macromolecular Reviews 1981, 16, $41-122$.

(7) Annable, T.; Buscall, R.; Ettelaie, R.; Whittlestone, D. J. Rheol. 1993, 37, 695-726.

(8) Kloxin, C. J.; Scott, T. F.; Adzima, B. J.; Bowman, C. N. Macromolecules 2010, 43, $2643-2653$.

(9) Montarnal, D.; Capelot, M.; Tournilhac, F.; Leibler, L. Science 2011, 334, 965-968. 
(10) Capelot, M.; Montarnal, D.; Tournilhac, F.; Leibler, L. J. Am. Chem. Soc. 2012, 134, $7664-7667$.

(11) Denissen, W.; Winne, J. M.; Du Prez, F. E. Chem. Sci. 2016, 7, 30-38.

(12) Brutman, J. P.; Delgado, P. A.; Hillmyer, M. A. ACS Macro Lett. 2014, 3, 607-610.

(13) Yang, Y.; Pei, Z.; Zhang, X.; Tao, L.; Wei, Y.; Ji, Y. Chem. Sci. 2014, 5, 3486-3492.

(14) Denissen, W.; Rivero, G.; Nicolaÿ, R.; Leibler, L.; Winne, J. M.; Du Prez, F. E. Adv. Funct. Mater. 2015, 25, 2451-2457.

(15) Obadia, M. M.; Mudraboyina, B. P.; Serghei, A.; Montarnal, D.; Drockenmuller, E. J. Am. Chem. Soc. 2015, 137, 6078-6083.

(16) Hendriks, B.; Waelkens, J.; Winne, J. M.; Du Prez, F. E. ACS Macro Lett. 2017, 6, 930-934.

(17) Canadell, J.; Goossens, H.; Klumperman, B. Macromolecules 2011, 44, 2536-2541.

(18) Michal, B. T.; Jaye, C. A.; Spencer, E. J.; Rowan, S. J. ACS Macro Lett. 2013, 2, 694-699.

(19) Fortman, D. J.; Brutman, J. P.; Cramer, C. J.; Hillmyer, M. A.; Dichtel, W. R. J. Am. Chem. Soc. 2015, 13\%, 14019-14022.

(20) Suzuki, A. J. Organomet. Chem. 1999, 576, 147-168.

(21) Roy, C. D.; Brown, H. C. J. Organomet. Chem. 2007, 692, 784-790.

(22) Niu, W.; O’Sullivan, C.; Rambo, B. M.; Smith, M. D.; Lavigne, J. J. Chem. Comm. 2005, 4342-4344.

(23) Röttger, M.; Domenech, T.; van der Weegen, R.; Breuillac, A.; Nicolä̈, R.; Leibler, L. Science 2017, 356, 62-65. 
(24) Cash, J. J.; Kubo, T.; Dobbins, D. J.; Sumerlin, B. S. Polym. Chem. 2018, 9, 20112020.

(25) Leibler, L.; Rubinstein, M.; Colby, R. H. Macromolecules 1991, 24, 4701-4707.

(26) Tanaka, F.; Edwards, S. Macromolecules 1992, 25, 1516-1523.

(27) Drozdov, A. D. Acta Mech. 1999, 133, 13-37.

(28) Rubinstein, M.; Semenov, A. N. Macromolecules 2001, 34, 1058-1068.

(29) Hui, C.-Y.; Long, R. Soft Matter 2012, 8, 8209-8216.

(30) Meng, F.; Pritchard, R. H.; Terentjev, E. M. Macromolecules 2016, 49, 2843-2852.

(31) Meng, F.; Terentjev, E. M. Macromolecules 2018, 51, 4660-4669.

(32) Jongschaap, R. J. J.; Wientjes, R. H. W.; Duits, M. H. G.; Mellema, J. Macromolecules 2001, 34, 1031-1038.

(33) Smallenburg, F.; Leibler, L.; Sciortino, F. Phys. Rev. Lett. 2013, 111, 188002.

(34) Ciarella, S.; Sciortino, F.; Ellenbroek, W. G. Phys. Rev. Lett. 2018, 121, 058003.

(35) Rovigatti, L.; Nava, G.; Bellini, T.; Sciortino, F. Macromolecules 2018, 51, 1232-1241.

(36) Tanaka, F.; Edwards, S. J. Non-Newton Fluid Mech. 1992, 43, 247-271.

(37) Meng, F.; Terentjev, E. M. Polymers 2016, 8, 108.

(38) Saed, M. O.; Torbati, A. H.; Starr, C. A.; Visvanathan, R.; Clark, N. A.; Yakacki, C. M. J. Polym. Sci. B: Polym. Phys. 2017, 55, 157-168.

(39) Chen, Y.; Tang, Z.; Zhang, X.; Liu, Y.; Wu, S.; Guo, B. ACS Appl. Mater. Interfaces 2018, 10, 24224-24231. 
(40) Cash, J. J.; Kubo, T.; Bapat, A. P.; Sumerlin, B. S. Macromolecules 2015, 48, 20982106.

(41) Cromwell, O. R.; Chung, J.; Guan, Z. J. Am. Chem. Soc. 2015, 13\%, 6492-6495.

(42) Cash, J. J.; Kubo, T.; Dobbins, D. J.; Sumerlin, B. S. Polym. Chem. 2018, 9, 20112020.

(43) Kolb, H. C.; Finn, M. G.; Sharpless, K. B. Angew. Chem. Intl. Ed. 2001, 40, 2004-2021.

(44) Scott, T. F.; Schneider, A. D.; Cook, W. D.; Bowman, C. N. Science 2005, 308, 16151617.

(45) Hoyle, C. E.; Bowman, C. N. Angew. Chem. Intl. Ed. 2010, 49, 1540-1573.

(46) Kade, M. J.; Burke, D. J.; Hawker, C. J. J. Polym. Sci. Part A: Polym. Chem. 2010, 48, 743-750.

(47) Hayashi, M.; Yano, R.; Takasu, A. Polym. Chem. 2019, 10, 2047-2056.

(48) Chen, X.; Li, L.; Wei, T.; Venerus, D. C.; Torkelson, J. M. ACS Appl. Mater. Interfaces 2019, 11, 2398-2407.

(49) Hotta, A.; Clarke, S. M.; Terentjev, E. M. Macromolecules 2002, 35, 271-277.

(50) Gent, A. Rubber Chem. Technol. 1996, 69, 59-61. 